\title{
Frequency-Dependent Glycinergic Inhibition Modulates Plasticity in Hippocampus
}

\author{
Tara Keck, Kyle P. Lillis, Yu-Dong Zhou, and John A. White \\ Department of Biomedical Engineering, Center for BioDynamics, Center for Memory and Brain, Boston University, Boston, Massachusetts 02215
}

Previous studies have demonstrated the presence of functional glycine receptors (GlyRs) in hippocampus. In this work, we examine the baseline activity and activity-dependent modulation of GlyRs in region CA1. We find that strychnine-sensitive GlyRs are open in the resting CA1 pyramidal cell, creating a state of tonic inhibition that "shunts" the magnitude of EPSPs evoked by electrical stimulation of the Schaffer collateral inputs. This GlyR-mediated shunting conductance is independent of the presynaptic stimulation rate; however, pairs of presynaptic and postsynaptic action potentials, repeated at frequencies above $5 \mathrm{~Hz}$, reduce the GlyR-mediated conductance and increase peak EPSP magnitudes to levels at least $20 \%$ larger than those seen with presynaptic stimulation alone. We refer to this phenomenon as rate-dependent efficacy (RDE). Exogenous GlyR agonists (glycine, taurine) block RDE by preventing the closure of postsynaptic GlyRs. The GlyR antagonist strychnine blocks postsynaptic GlyRs under all conditions, occluding RDE. During RDE, GlyRs are less responsive to local glycine application, suggesting that a reduction in the number or sensitivity of membrane-inserted GlyRs underlies RDE. By extending the RDE induction protocol to include 500 paired presynaptic and postsynaptic spikes, we can induce long-term synaptic depression (LTD). Manipulations that lead to reduced functionality of GlyRs, either pharmacologically or through $\mathrm{RDE}$, also lead to increased LTD. This result suggests that RDE contributes to long-term synaptic plasticity in the hippocampus.

Key words: glycine; long-term depression; CA1; hippocampus; frequency; patch clamp

\section{Introduction}

GABA is often assumed to be the sole inhibitory neurotransmitter in the hippocampus. Recent evidence has suggested that glycine, the other major inhibitory neurotransmitter of the CNS, serves as a neurotransmitter in hippocampus as well. First, mRNA for glycine receptors (GlyRs) is expressed in hippocampal pyramidal cells (PCs) (Racca et al., 1998). Second, focal application of glycine evokes strychnine-sensitive outward currents in CA1 PCs (Chattipakorn and McMahon, 2002). Third, immunocytochemical studies provide direct anatomical evidence that hippocampus is innervated by glycinergic terminals (Danglot et al., 2004; Zeilhofer et al., 2005; Song et al., 2006). Other recent work has demonstrated that glycinergic synapses innervate the proximal apical dendrites of CA1 PCs in stratum radiatum. These glycinergic terminals arise, at least in part, from GABAergic interneurons (Danglot et al., 2004; Song et al., 2006). Whereas the source of glycine in the hippocampus remains unclear,

\footnotetext{
Received Sept. 27, 2006; revised April 10, 2008; accepted May 27, 2008.

This work was supported by grants from the National Institutes of Health. We thank P. B. Cook for important insights related to the interpretation of the data and M. E. Hasselmo, C. Leibold, N. A. Lesica, J.-W. Lin, V. Scheuss, and V. Stein for helpful discussions and comments on this manuscript.

The authors declare no competing financial interests.

Correspondence should be addressed to either of the following at their present address: Dr. Tara Keck, Department of Cellular and Systems Neurobiology, Max Planck Institute of Neurobiology, Am Klopferspitz 18, D-82152 Martinsried, Germany, E-mail: keck@neuro.mpg.de; or Dr. John A. White, Department of Bioengineering, University of Utah, 20 South 2030 East, 108 BPRB, Salt Lake City, UT 84112, E-mail: john.white@utah.edu.

Y.-D. Zhou's present address: Department of Neurology, Beth Israel Deaconess Medical Center and Harvard

Medical School, Harvard Institutes of Medicine, 77 Avenue Louis Pasteur, Boston, MA 02115.

DOI:10.1523/JNEUROSCI.5618-07.2008

Copyright $\odot 2008$ Society for Neuroscience $\quad 0270-6474 / 08 / 287359-11 \$ 15.00 / 0$
}

calbindin-, calretinin-, and parvalbumin-positive interneurons in CA1 are hypothesized to corelease GABA and glycine (Danglot et al., 2004), which are carried by the same vesicular transporter (Wojcik et al., 2006).

Glycine in the synaptic cleft is primarily regulated by type 1 and type 2 glycine transporters (GlyT1 and GlyT2), which transport glycine back into glia (GlyT1) or the presynaptic terminals (GlyT2) (Jursky and Nelson, 1995). Changes in GlyT1 activity have been shown to alter both glutamatergic transmission (Martina et al., 2005) and long-term potentiation (LTP) (Martina et al., 2004), in a manner that is hypothesized to depend on the role of glycine as a coagonist of NMDA receptors. GlyT2 is expressed by calbindin-positive GABAergic neurons in CA1 (Danglot et al., 2004) and under physiological conditions transports glycine into, but not out of, the presynaptic cell (Roux and Supplisson, 2000). In the hippocampus, little work has been done investigating the role of GlyT2 transporters on either transmission or plasticity, and no work has examined effects of GlyR activity on long-term synaptic depression (LTD).

Here, we show that a proportion of strychnine-sensitive GlyRs are open in the resting cell, creating a state of tonic inhibition that appears to "shunt" EPSPs evoked by shocking the Schaffer collaterals, decreasing EPSP peak magnitude. With presynaptic stimulation alone, glycinergic shunting of EPSP magnitude is independent of the synaptic stimulation rate and presynaptically mediated short-term facilitation. In contrast, with paired postsynaptic action potentials (APs) and presynaptic EPSP stimulation at frequencies higher than $5 \mathrm{~Hz}$, postsynaptic GlyRs close and peak EPSP magnitude increases substantially beyond what is observed for presynaptic facilitation of EPSPs alone. We refer to 
this phenomenon as rate-dependent efficacy (RDE). Responses to pressure-ejected glycine are decreased during RDE, suggesting that RDE is mediated through changes to the GlyRs themselves (either through reduced receptor sensitivity or receptor trafficking that reduces the number of available receptors). Finally, we observe that the same conditions that lead to RDE also lead to an increase in LTD induced by low-frequency pairing of presynaptic and postsynaptic activity.

\section{Materials and Methods}

Hippocampal slice preparation. All experiments were conducted as approved by the Boston University Institutional Animal Care and Use Committee. Long-Evans rats (in a subset of the experiments, Wistar rats were used), aged 14-21 d, were anesthetized with isoflurane and decapitated. The brain was removed, placed in chilled $\left(4^{\circ} \mathrm{C}\right)$ artificial CSF (ACSF; see below, Solutions and drugs), and sliced using a vibratome to $300 \mu \mathrm{m}$ thickness. After the dissection, slices were allowed to recover at $35^{\circ} \mathrm{C}$ for $30 \mathrm{~min}$ in a holding chamber and were brought back to room temperature for at least $30 \mathrm{~min}$ before recording. The slices were perfused in heated $\operatorname{ACSF}\left(35^{\circ} \mathrm{C}\right)$ and equilibrated with $95 \% \mathrm{O}_{2} / 5 \% \mathrm{CO}_{2}$ ACSF in a separate holding chamber (Warner Instruments) mounted on a microscope stage.

Solutions and drugs. For both the dissection and experiments, the slices were bathed in ACSF (in mm: $126 \mathrm{NaCl}, 1.25 \mathrm{NaH}_{2} \mathrm{PO}_{4}, 2 \mathrm{MgCl}, 26$ $\mathrm{NaHCO}_{3}, 10$ glucose, $2.5 \mathrm{KCl}$, and $\left.2 \mathrm{CaCl}_{2}\right)$. For experiments with 6-cyano-7-nitroquinoxaline-2,3-dione (CNQX) in the bath, we used ACSF with $0.2 \mathrm{~mm} \mathrm{MgCl}$, instead of $2 \mathrm{~mm} \mathrm{MgCl}$. For all recordings, the pipette contained the following intracellular recording solution: $120 \mathrm{mM}$ K-gluconate, $10 \mathrm{~mm} \mathrm{KCl,} 10 \mathrm{~mm}$ HEPES, 4 mм Mg-ATP, 0.3 mм TrisGTP, $10 \mathrm{~mm} \mathrm{Na}_{2}$-phospocreatine, and $20 \mathrm{U}$ of creatine kinase, $\mathrm{pH} 7.2$, with $\mathrm{KOH}$. In the experiments using 1,2-Bis(2-aminophenoxy)ethane$N, N, N^{\prime}, N^{\prime}$-tetraacetic acid (BAPTA) and 4-aminopyridine (4-AP), the appropriate amount of drug was added to the above intracellular solution. All other drugs were made in stock solutions and stored in frozen aliquots ( $100 \mu \mathrm{l}$ to $1 \mathrm{ml}$ ), which were dissolved into $100 \mathrm{ml}$ of ACSF on the day of the experiment. All agents were purchased from Sigma.

Electrophysiology. Pyramidal neurons in CA1 were identified visually and recorded with whole-cell patch-clamp recording techniques using infrared differential interference contrast visualization (Axioskop 2 FS; Carl Zeiss). Recordings were made using borosilicate microelectrodes (3-5 $\mathrm{M} \Omega, 1.0$ outer diameter, 0.75 inner diameter; Sutter Instrument) filled with intracellular recording solution. Resting membrane potential was recorded immediately after breaking into the cell (average, $-64.6 \pm$ $0.32 \mathrm{mV} ; n=348)$. Input resistance, access resistance, and resting membrane potential were monitored, and in the case of significant changes $\left(\Delta R_{\mathrm{i}}>20 \% ; \Delta R_{\mathrm{a}}>20 \% ; \Delta V_{\mathrm{r}}>10 \%\right)$ that were not coincident with changes in the pharmacological environment, cells were discarded. A stimulation electrode (1-3M $\Omega$ ) filled with ACSF and placed in the Schaffer collateral layer, at least $300 \mu \mathrm{m}$ from the recorded cell, was used to elicit EPSPs at the proximal dendrite. Stimulus intensity, in the range of 5-35 $\mu \mathrm{A}$ for $500 \mu \mathrm{s}$, was adjusted to induce EPSPs with a magnitude of 2-4 $\mathrm{mV}$ and limited EPSP failures. Somatic recordings were made under current-clamp conditions at resting potential, and voltage changes were measured in the soma.

Measuring RDE. For all experiments testing RDE, 25 pairs of AP followed by an EPSP after a delay were averaged, the AP was subtracted out using a baseline AP measurement, and the resulting peak EPSP magnitude was measured and normalized by the baseline peak EPSP magnitude (the peak magnitude measured in the absence of the AP at $0.5 \mathrm{~Hz}$ ). These experiments were performed with a constant delay (ranging from 10 to $60 \mathrm{~ms}$ ) between the AP and the EPSP. The above protocol was measured first in control ACSF with $10 \mu \mathrm{M}$ bicuculline in the bath and then repeated as one of the following blockers was bath applied (in addition to the $10 \mu \mathrm{M}$ bicuculline): $50 \mu \mathrm{M}$ DL-2-amino-5-phosphonopentanoic acid (APV), $10 \mu \mathrm{M}$ CNQX, $10 \quad \mu \mathrm{M}$ nifedipine, $15 \mu \mathrm{M}$ 1,2,5,6tetrahydropyridin-4-yl methylphosphinic acid (TPMPA), $20 \mu \mathrm{M}$ 4-ethylphenylamino-1,2-dimethyl-6-methylaminopyrimidinium chloride (ZD7288), $10 \mu \mathrm{M}$ picrotoxin, $0.01-10 \mu \mathrm{M}$ strychnine, $100 \mu \mathrm{M}$ amox- apine, 0.01-10 mM glycine, $1 \mathrm{~mm}$ taurine, $10 \mu \mathrm{M}$ 2-(3-carboxypropyl)-3amino-6-(4-methoxyphenyl) pyridazinium bromide gabazine (SR 95531), $1 \mu \mathrm{M}$ 1-(2,4-dichlorophenyl)-5-(4-iodophenyl)-4-methyl- $N$-1piperdinyl-1 $H$-pyrazole-3-carboxamide trifluoroacetate salt (AM 251), $20 \mu \mathrm{M} N \omega$-nitro-L-arginine methyl ester hydrochloride (L-NAME), 500 nм $N$-[3-(4'-fluorophenyl)-3-(4'-phenylphenoxy)propyl] sarcosine hydrochloride (ALX-5407), or $10 \mu \mathrm{M}$ ritanserin. All drugs were bath applied for 10 min before measurements were made, except ritanserin, which was bath applied for $1 \mathrm{~h}$, as was done in previous studies (Krause and Jia, 2005). In the following cases, drugs were applied intracellularly for $10 \mathrm{~min}$ before recordings were made: $10 \mu \mathrm{M}$ intracellular 4-AP and 5-20 mm intracellular BAPTA.

Paired-pulse facilitation was measured in both ASCF and strychnine using the standard protocol of two pulses elicited by the stimulation electrode at a constant delay (15-40 ms), repeated 25 times at $10 \mathrm{~Hz}$. To measure the magnitude of the second EPSP, the single EPSP alone measured at $10 \mathrm{~Hz}$ was subtracted from the second of the EPSPs to correct for the fact that the second EPSP occurs before the first EPSP ends. Excitability was measured as the firing rate resulting from $500 \mathrm{~ms}$ current pulses ranging from below threshold for each cell to $800 \mathrm{pA}$. AP threshold was measured as the minimal current step input $(500 \mathrm{~ms})$ necessary to elicit one AP. Conductance was calculated in voltage clamp, by measuring the current change resulting from $5 \mathrm{mV}$ hyperpolarizing voltage steps. Coefficient of variation (CV) analysis was calculated by dividing the SD of the peak EPSP magnitude by the mean of the peak EPSP magnitude in each of the specified conditions.

For experiments measuring evoked responses to locally applied glycine, we placed an electrode $(3-5 \mathrm{M} \Omega$ ) filled with $300 \mu \mathrm{M}$ glycine $\sim 50$ $\mu \mathrm{m}$ from the soma, near the apical dendrites. Currents evoked from the pressure application ( $3 \mathrm{psi}$ ) of glycine using a picospritzer were measured in current clamp. The duration of the pressure pulses were adjusted $(50-300 \mathrm{~ms})$ so that the evoked current did not lead to action potentials. Glycine-evoked currents were measured (1) when the cell was at rest, (2) $50 \mathrm{~ms}$ after 25 repetitions of $10 \mathrm{~Hz}$ AP-EPSP pairs $(20 \mathrm{~ms}$ interval between the AP and EPSP), and (3) $50 \mathrm{~ms}$ after 25 repetitions of $1 \mathrm{~Hz}$ AP-EPSP pairs (20 ms interval between the AP and EPSP). The three conditions were interleaved, with $20 \mathrm{~s}$ between trials.

For LTD experiments, baseline EPSP magnitude was measured at 0.1 $\mathrm{Hz}$ for $10 \mathrm{~min}$. Conditioning pulses (CPs; a postsynaptic AP followed by a presynaptic stimulated EPSP after a 20 ms delay) were given at 1 or 10 $\mathrm{Hz}$ and repeated 500 times. Changes in the post-CP EPSP magnitude were measured at $0.1 \mathrm{~Hz}$ for $20-30 \mathrm{~min}$, beginning immediately after the CPs. All LTD experiments were done with $10 \mu \mathrm{M}$ bicuculline in the bath (to block $\mathrm{GABA}_{\mathrm{A}}$ receptors), as well as other specified drugs (see Fig. 6).

Data acquisition, analysis, and statistics. Recordings were made using a MultiClamp 700A amplifier (Molecular Devices), controlled by custom software written in pClamp 8.0 (Molecular Devices). Samples were acquired at $10 \mathrm{kHz}$ and filtered at $1 \mathrm{kHz}$ using a Bessel filter. Pipette offset was corrected for using the MultiClamp Commander. The liquid junction potential was not corrected; calculations found it to be $\sim 10 \mathrm{mV}$. To address the potential access or series resistance issues in these recordings, the bridge was balanced at the beginning of the experiment and then periodically throughout to make sure that the recording was stable. All data were analyzed after recordings using custom protocols written in Matlab (MathWorks).

All population data were expressed as the mean \pm SEM. Statistical tests were paired $t$ tests, a $t$ test followed by a correlation coefficient calculation, an ANOVA, or $t$ test, followed by Bonferroni's multiple comparison tests, as indicated in the text.

\section{Results \\ Characterizing GlyRs in hippocampus}

We studied the basic properties of postsynaptic GlyRs known to be present in hippocampus (Racca et al., 1998; Chattipakorn and McMahon, 2002). First, we investigated whether these receptors contribute to the resting membrane conductance of CA1 PCs under whole-cell voltage clamp. By applying hyperpolarizing voltage steps, we measured the resulting current and calculated 
a

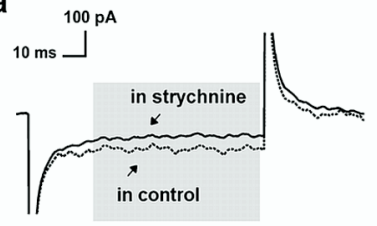

b $100 \mathrm{pA}$
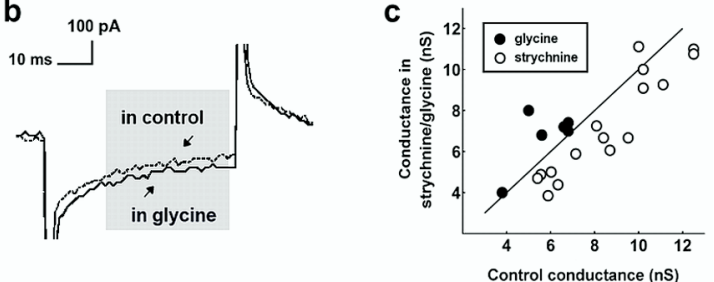

d

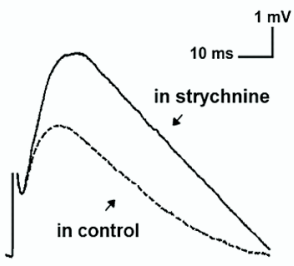

e

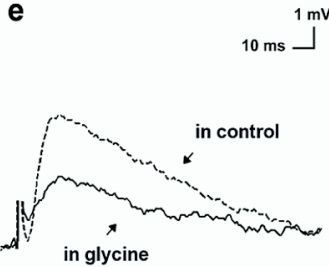

f

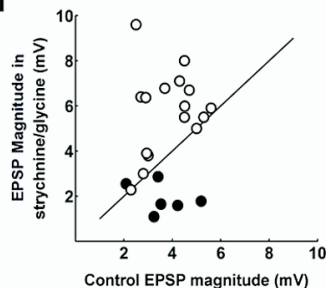

g
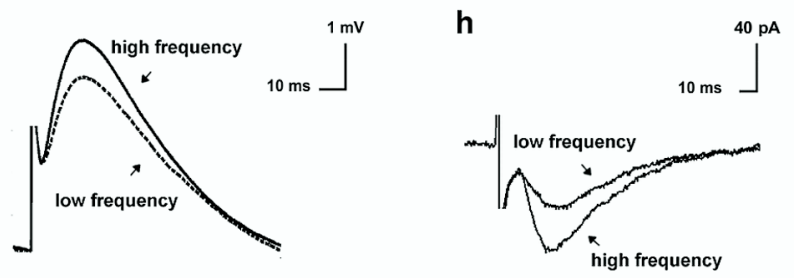

i

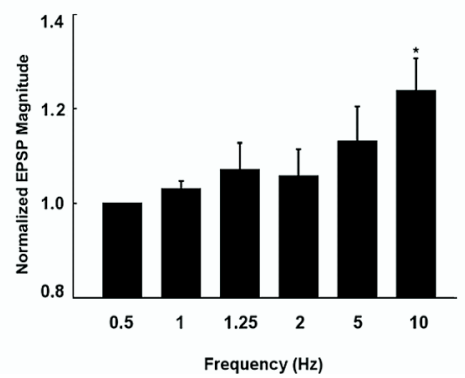

j

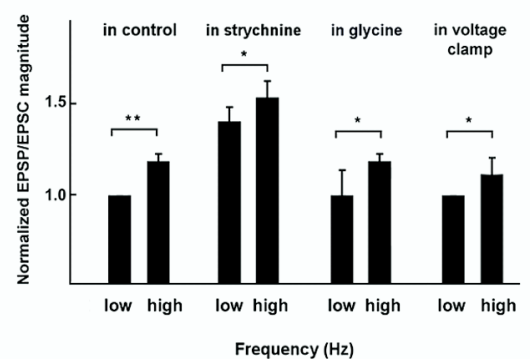

Figure 1. GlyRs create a state of tonic inhibition in hippocampal CA1 neurons. $\boldsymbol{a}, \boldsymbol{b}$, Sample trace of cellular conductance measured in voltage clamp with a $5 \mathrm{mV}$ hyperpolarizing step, in control solution (dashed line), strychnine (a, solid line; $1 \mu \mathrm{m}$ ), or glycine ( $\boldsymbol{b}$, solid line; $10 \mathrm{~mm}$ ). The gray box represents the steady-state component of the signal, from which we measured the conductance. $c$, Conductance measured in control solutions versus the conductance measured in strychnine (open circles; $10 \mu \mathrm{M}$ strychnine, $n=8 ; 1 \mu \mathrm{m}$ strychnine, $n=8$; paired $t$ test, strychnine vs control: $p=0.0004, n=16$ ) or glycine (filled circles; 10 mm; paired $t$ test, glycine vs control: $p<0.05, n=6$ ). $\boldsymbol{d}$, EPSPs were elicited on the proximal dendrite without evoking postsynaptic APs. In the sample traces, the dashed line shows the EPSP in ACSF, and the solid line shows the EPSP with the GlyR blocker strychnine $(1 \mu \mathrm{m})$ bath applied. $e$, Sample traces. The dashed line shows the EPSP in ACSF, and the solid line shows the EPSP in glycine (10 mM). $f$, Peak EPSP magnitude measured in control solutions versus the peak EPSP magnitude measured in strychnine (open circles; $10 \mu \mathrm{m}$ strychnine, $n=8 ; 1 \mu \mathrm{m}$ strychnine, $n=8$; paired $t$ test, strychnine vs control: $p<0.05, n=16$ ) or 10 mm glycine (filled circles; paired $t$ test, glycine vs control: $p<0.05, n=6) . \boldsymbol{g}, \boldsymbol{h}$, Raw data traces of evoked EPSP $(\boldsymbol{g})$ and EPSC (h) alone, repeated in trains at low $(0.5 \mathrm{~Hz})$ and high $(10 \mathrm{~Hz})$ frequencies in current clamp $(\boldsymbol{g})$ and voltage clamp $(\boldsymbol{h})$. $\boldsymbol{i}$, Average \pm SEM over 25 repetitions of peak EPSP magnitude as a function of trial frequency for EPSPs alone. Peak EPSP magnitudes were normalized by the peak EPSP magnitude recorded in the absence of APs at $0.5 \mathrm{~Hz}$ (baseline EPSP). EPSP increases with stimulation frequency ( $10 \mathrm{~Hz}$ vs $0.5 \mathrm{~Hz}$ EPSP; Bonferroni's multiple comparison test, ${ }^{*} p<0.05 ; n=14$ ). $\boldsymbol{j}$, Normalized peak EPSP magnitudes for EPSP alone at low $(0.5-1 \mathrm{~Hz})$ and high $(5-10 \mathrm{~Hz})$ frequencies are different in control (paired $t$ test, $\left.{ }^{*} p<0.05 ; n=150\right)$, strychnine (paired $t$ test, ${ }^{*} p<0.05 ; 1 \mu \mathrm{m}$ strychnine, $n=8 ; 10 \mu \mathrm{m}$ strychnine, $n=8$ ), glycine (paired $t$ test, ${ }^{*} p<0.05 ; 10 \mathrm{mm;}$ $n=6$ ), or voltage clamp [normalized value, 1.14 at $10 \mathrm{~Hz}$ (normalized by EPS ( peak magnitude at $0.5 \mathrm{~Hz}$ ); two-way ANOVA: 0.5 $\mathrm{Hz}, 10 \mathrm{~Hz}$, EPSP and EPSC; low vs high frequency, $p=0.04$; EPSC vs EPSP, $p=0.45 ; n=6]$.

the input conductance of the cell. With the bath application of the GlyR antagonist strychnine $(1 \mu \mathrm{M})$, we measured a significant decrease in the steady-state input conductance, as illustrated in the sample trace shown in Figure $1 a$. In 15 of the 16 cells measured, the conductance of the cell decreased with the application of strychnine (Fig. 1c, open circles). Not surprisingly, the mean value of input conductance was changed significantly in strychnine (normalized value, $0.833 \pm 0.03$; mean \pm SEM; $p<0.01$ ).

With the bath application of the endogenous GlyR agonist glycine (10 $\mathrm{mm})$, we measured an increase in the steady-state input conductance, as shown in a sample trace in Figure $1 b$. In all six of the cells measured, the conductance increased with the bath application of glycine (Fig. 1c, filled circles), and the mean conductance measured in glycine was increased compared with control (normalized value, $1.17 \pm 0.08 ; p<0.05)$. These data suggest that a proportion of GlyRs are open in the quiescent slice, as evidenced by the conductance change measured in strychnine; however, these receptors are not saturated, because bath-applied glycine increases the measured conductance.

As was suggested previously by other groups (Song et al., 2006; Zhang et al., 2006), we sought to demonstrate that the resting glycinergic conductance affects EPSP amplitude. By stimulating the Schaffer collaterals, we evoked $0.5 \mathrm{~Hz}$ trains of 25 EPSPs in the stratum radiatum of CA1 PCs and measured the subsequent EPSPs in the soma, while recording in current clamp. With the bath application of the GlyR antagonist strychnine $(1 \mu \mathrm{M})$, we observed a large increase in the peak EPSP magnitude, as shown for a typical cell in Figure $1 d$. Across a sample of 16 cells, the peak EPSP magnitude increased in 14 of 16 cells in strychnine versus control ACSF (Fig. 1f, open circles) (normalized value, $1.4 \pm 0.08 ; p<0.01$ ). The degree of change in the peak EPSP magnitude was dependent on the concentration of strychnine, with concentrations as low as $100 \mathrm{nM}$ completely blocking the glycinergic shunting of the EPSP (supplemental Fig. S1 $a$, available at www.jneurosci.org as supplemental material). With bath application of glycine $(10 \mathrm{~mm})$, we measured a decrease in the peak EPSP magnitude, shown in an example trace in Figure 1e. The EPSP magnitude was decreased with glycine application (10 $\mathrm{mM}$ ) in five of six cells (Fig. $1 f$, filled circles), and the mean peak EPSP magnitude after the glycine application was significantly decreased compared with the mean peak EPSP magnitude measured in control solution (normalized value, $0.59 \pm 0.14$; $p<0.05)$. The decrease in peak EPSP magnitude was dependent on the concentration of glycine (supplemental Fig. S1b, available at www.jneurosci.org as supplemental material).

Together, these data suggest that a proportion of the GlyRs are open in the quiescent slice, creating a state of tonic inhibitory shunt. When these GlyRs are blocked through the application of strychnine, this glycinergic inhibition is reduced, and the peak EPSP magnitude is increased. Furthermore, glycine application increases conductance and the glycinergic shunt, thereby decreasing the EPSP magnitude. These data indicate that although a 
significant proportion of GlyRs are open in the resting slice, the GlyRs are not saturated.

\section{RDE: phenomenology}

To determine the possible role of this glycinergic inhibition in the hippocampus, we investigated whether this glycinergic inhibition depends on cellular activity. First, we established the effects of stimulation frequency on the peak EPSP magnitude in the control conditions (i.e., in the absence of glycinergic agonists or antagonists). As has been described previously (for review, see Zucker and Regehr, 2002; Abbott and Regehr, 2004), presynaptic stimulation elicited facilitation, the magnitude of which increased with increasing stimulation frequency (Fig. 1i). Across a sample of 150 cells, the mean peak EPSP magnitude measured during trains of 25 EPSPs at a stimulation frequency of $10 \mathrm{~Hz}$ was significantly larger (normalized value, $1.2 \pm$ 0.08 ) than that measured at a frequency of $0.5 \mathrm{~Hz}$ (Fig. 1g,j, control). We found that neither strychnine nor glycine altered presynaptic facilitation (Fig. 1j), such that in both glycine and strychnine, the highfrequency $(5-10 \mathrm{~Hz})$ EPSPs are larger than the low-frequency $(0.5-1 \mathrm{~Hz})$ EPSPs. The degree of facilitation is not changed if measurements are made under voltage clamp of the postsynaptic cell (Fig. 1h,j, voltage clamp): in a two-way ANOVA, we saw a significant effect of stimulation frequency $(p=0.04)$, but not of measurement mode (current clamp vs voltage clamp; $p=0.45$; $n=6)$. Because voltage-clamp measurements reduce the number of postsynaptic variables, these results suggest that this facilitation is primarily presynaptic.

Next, we examined EPSP magnitude in response to paired postsynaptic and presynaptic activity, with the postsynaptic AP leading the presynaptically stimulated EPSP in trains of 25 AP-EPSP pairs. We found a frequency-dependent increase in peak EPSP magnitude, as shown for a sample cell in Figure $2 a$ and for the population in Figure $2 b$ (open bars). Over a sample of 12 cells, the mean peak EPSP magnitude for AP-EPSP pairs at $10 \mathrm{~Hz}$ was significantly larger (normalized value, $1.4 \pm$ 0.1 ) than that for $0.5 \mathrm{~Hz}$ AP-EPSP pairs (Fig. 2b). The increase in peak EPSP magnitude observed for AP-EPSP pairs at $10 \mathrm{~Hz}$ was also significantly larger than the presynaptic facilitation observed at the same frequency (Fig. $2 c$, first and second bars). We refer to this additional frequency-dependent increase in peak EPSP magnitude elicited by AP-EPSP pairs as RDE. This effect required repeated pairing of APs with EPSPs, because a single AP-EPSP pair did not evoke either facilitation or RDE (Fig. $2 c$ ), and the peak EPSP magnitudes measured after a train of high-frequency $(10 \mathrm{~Hz})$ postsynaptic APs alone were not significantly different from low-frequency EPSPs alone (Fig. 2c). We additionally tested whether RDE depended on the interval between the postsynaptic $\mathrm{AP}$ and the presynaptically stimulated EPSP. We found that the ratio of the peak EPSP magnitude measured during high- frequency AP-EPSP pairs to the peak EPSP magnitude measured during low-frequency AP-EPSP pairs was constant for intervals ranging from 10 to $60 \mathrm{~ms}$ (supplemental Fig. S2, available at www.jneurosci.org as supplemental material). These results indicate that whereas trains of presynaptic and postsynaptic activity are necessary to induce RDE, the exact timing is not important.

\section{RDE is caused by the closure of postsynaptic GlyRs}

To determine whether the EPSP magnitude increases observed during RDE were attributable to modulation of glycinergic inhibition, we measured the effect of strychnine $(0.01-10 \mu \mathrm{M})$ and high concentrations of exogenous glycine $(1 \mathrm{~mm})$ on RDE and found that RDE does not occur when either strychnine or glycine is bath applied. More specifically, in the presence of strychnine, the peak EPSP magnitude measured during AP-EPSP pairs at low frequency $(0.5-1 \mathrm{~Hz})$ increases to the peak EPSP magnitude observed for the high-frequency $(5-10 \mathrm{~Hz})$ AP-EPSP pairs, as shown for an example cell in Figure $3 a$. Across a sample of 16 cells in strychnine, the mean peak EPSP magnitude during EPSPs alone at high frequency increases, so that it is no longer different from that measured during AP-EPSP pairs at high frequency 
a

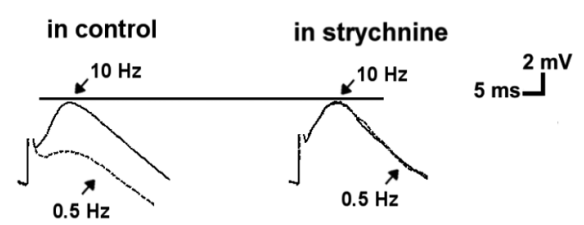

b

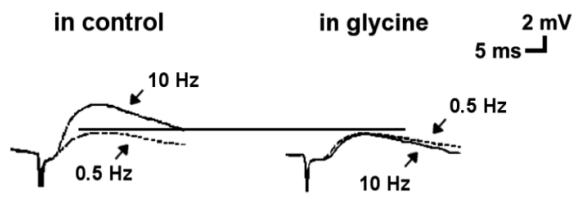

C

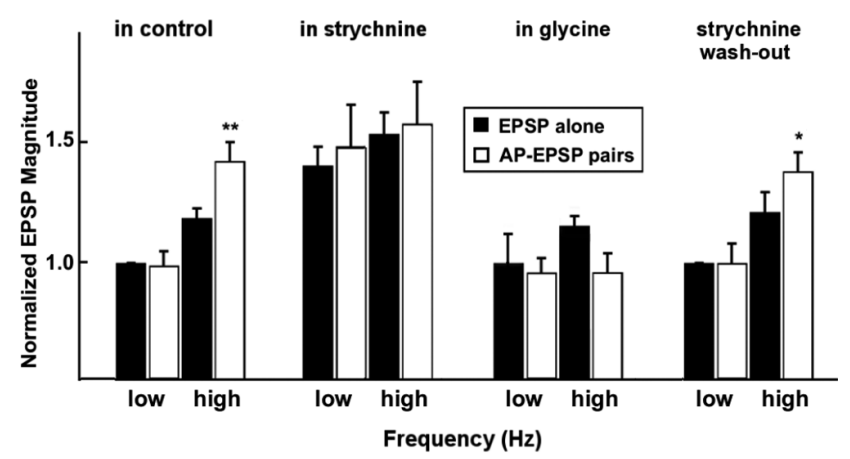

d

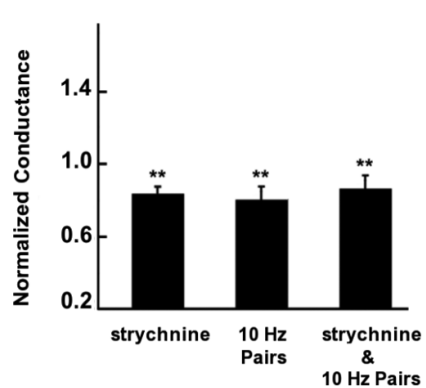

Figure 3. Closure of GlyRs is responsible for RDE. $\boldsymbol{a}$, Strychnine occludes RDE. The dashed line represents the EPSP at low frequency, and the solid line represents the EPSP at high frequency (from AP-EPSP pairs; AP not shown). EPSPs are shown in control solution (left) and in strychnine (right). The EPSP at low frequency increases to the same size as the high-frequency case in the presence of strychnine $(1 \mu \mathrm{m}) . \boldsymbol{b}$, Glycine prevents RDE. The dashed line represents the EPSP at low frequency, and the solid line represents the EPSP athigh frequency (from AP-EPSP pairs; AP notshown). EPSPs are shown in control solution (left) and in glycine (right). The EPSP athigh frequency decreases to the same size as the low-frequency case in the presence of glycine (1 mM). $c$, Normalized peak EPSP magnitudes for AP-EPSP pairs (open bars) and EPSPs alone (filled bars) as a function of both the bath solutions and the firing rate. AP-EPSP pairs at high frequency are significantly different from EPSPs alone at high frequency in control solution (paired $t$ test, $p=3 \times 10^{-6} ; n=24$ ), but not in strychnine [paired $t$ test, $p=0.62 ; n=16$ (10 $\mu$ m strychnine, $n=8 ; 1 \mu \mathrm{m}$ strychnine, $n=8)$ ] or glycine (paired $t$ test, $p>0.7 ; n=13 ; 1 \mathrm{~mm}$ glycine). AP-EPSP pairs at low frequency are not significantly different from EPSPs alone at low frequency in any solutions [Bonferroni's multiple comparison test: control solution, $p=0.64, n=24 ;$ strychnine, $p=0.22, n=16(10 \mu$ m strychnine, $n=8 ; 1 \mu$ m strychnine, $n=8)$; glycine, $p=0.52, n=13 ; 1$ mm glycine]. The occlusion of RDE by strychnine is reversible. After strychnine is washed out for $25 \mathrm{~min}$, RDE returns (paired ttest, $p=0.02 ; 10 \mathrm{HzAP}-$ EPSP pairs vs $10 \mathrm{~Hz}$ EPSP alone in strychnine washout). $\boldsymbol{d}$, Conductance, normalized by conductance measured in ACSF, measured with the bath application of strychnine, in the first $50 \mathrm{~ms}$ after AP-EPSP pairs in control solutions, or immediately after RDE in strychnine is significantly smaller than conductance measured at rest in control solutions [paired $t$ test: strychnine, $p=0.0004, n=16(10 \mu \mathrm{m}$ strychnine, $n=8 ; 1$ $\mu$ m strychnine, $n=8)$; RDE, $p=0.005, n=7$; RDE and strychnine, $p<0.01, n=8]$, Strychnine or RDE conductances are not different from one another (Bonferroni's multiple comparison test, $p=0.66$ ). Data are mean \pm SEM. ${ }^{*} p<0.05 ;{ }^{* *} p<0.01$.
(Fig. 3c). This effect is reversible (Fig. 3c, washout). This result suggests that strychnine occludes RDE by shutting down GlyRs. Because strychnine blocks a small percentage of $\mathrm{GABA}_{\mathrm{A}}$ receptors at concentrations of $>1 \mu \mathrm{M}$ ( $\mathrm{Li}$ et al., 2003), we tested the dependence of RDE on strychnine concentration. We found that strychnine prevents RDE completely at concentrations as low as $100 \mathrm{~nm}$ (supplemental Fig. S3, available at www.jneurosci.org as supplemental material) (paired $t$ test, $p=0.48 ; n=4 ; 10 \mathrm{~Hz}$ EPSP alone vs $10 \mathrm{~Hz}$ AP-EPSP pairs in $100 \mathrm{~nm}$ strychnine). To further confirm these findings, we tested the effects of picrotoxin on RDE. Picrotoxin is a less specific antagonist, because it blocks only a subset of the GlyRs and also blocks $\mathrm{GABA}_{\mathrm{A}}$ and $\mathrm{GABA}_{\mathrm{C}}$ receptors (Pribilla et al., 1992). In picrotoxin, we found similar effects to those in strychnine: the peak magnitude of EPSPs alone at $10 \mathrm{~Hz}$ increased to the same peak EPSP magnitude measured during AP-EPSP pairs at $10 \mathrm{~Hz}$ (paired $t$ test, $p=0.5 ; n=19$; data not shown).

In addition to strychnine and picrotoxin, we measured the effects of high concentrations of glycine (which has no effect on GABA receptors) on RDE. Complementary to our strychnine and picrotoxin results, we find that the bath application of high concentrations of glycine ( $1 \mathrm{~mm}$ ) eliminates RDE by preventing the closure of GlyRs. In these experiments, the mean peak magnitude of EPSPs for high-frequency AP-EPSP pairs is reduced to that of the low-frequency AP-EPSP pairs, shown in a sample trace in Figure $3 b$. Across a population of 13 cells, we found that the peak EPSP magnitude measured during $10 \mathrm{~Hz}$ AP-EPSP pairs decreases to the same peak magnitude as EPSPs alone at $10 \mathrm{~Hz}$ (Fig. $3 c$ ). We also studied the effects of taurine, a lower-affinity (yet specific) GlyR agonist, on RDE. In taurine, we found effects similar to those measured in exogenous glycine. The peak EPSP magnitude of AP-EPSP pairs at $10 \mathrm{~Hz}$ decreased to the same peak magnitude measured for EPSPs alone at $10 \mathrm{~Hz}$ (paired $t$ test, $p=$ $0.18 ; n=4$; data not shown).

Additional experiments revealed that the conductance change measured in the resting cell with the bath application of strychnine decreases by the same amount observed during highfrequency pairing in control solutions (Fig. $3 d$ ). This conductance change measured immediately following high-frequency AP-EPSP pairs is transient, lasting $<100 \mathrm{~ms}$ after pairing has ended. The conductance change measured during the coincident application of $10 \mathrm{~Hz}$ AP-EPSP pairs and strychnine is not larger than the conductance change measured for either AP-EPSP pairs or strychnine alone. These results suggest that paired activity induces RDE by closing the tonically open GlyRs. Extracellular glycine and taurine prevent RDE, suggesting that high concentrations of glycinergic agonists can overcome the tendency of GlyRs to close during high-frequency paired presynaptic and postsynaptic activity. Strychnine and picrotoxin apparently occlude RDE by blocking the postsynaptic GlyR-mediated conductance under all circumstances.

As an additional control, we measured the effects of specific $\mathrm{GABA}_{\mathrm{A}}$ receptor antagonists bicuculline (paired $t$ test, $p=0.01$; $n=5$; AP-EPSP pairs at $10 \mathrm{~Hz}$ vs EPSP alone at $10 \mathrm{~Hz}$ ) and SR 95531 (paired $t$ test, $p=0.03 ; n=4$; AP-EPSP pairs at $10 \mathrm{~Hz}$ vs EPSP alone at $10 \mathrm{~Hz}$ ) on RDE. In both of these antagonists, RDE persisted, suggesting that RDE is independent of $\mathrm{GABA}_{\mathrm{A}}$ receptors. A number of other bath-applied agonists and antagonists had no effect on RDE (all $t$ tests compare AP-EPSP pairs at high frequency vs low frequency, with $p<0.05$ indicating that RDE persists): $15 \mu \mathrm{M}$ TPMPA ( $\mathrm{GABA}_{\mathrm{C}}$ receptors; $\left.n=4 ; p=0.04\right), 10$ $\mu \mathrm{M} 4$-AP $\left(\mathrm{K}^{+}\right.$channels; $\left.n=9 ; p=0.001\right), 10 \mu \mathrm{M}$ nifedipine (L-type calcium channels; $n=6 ; p=0.02), 20 \mu \mathrm{M}$ ZD7288 (HCN 
channels; $n=7 ; p=0.05$ ), $10 \mu \mathrm{M}$ CNQX (in low $\left[\mathrm{Mg}^{2+}\right]$, AMPA receptors; $n=6 ; p=0.004), 50 \mu \mathrm{M}$ APV (NMDA receptors; $n=$ $8 ; p=0.004$ ), $20 \mu \mathrm{M} \mathrm{L}-\mathrm{NAME}$ (nitric oxide antagonist; $n=8 ; p=$ 0.007), AM 251 [endocannabinoid type 1 (CB1) antagonist; $n=5 ; p=0.03$ ], and $10 \mu \mathrm{M}$ ritanserin (blocks $5-\mathrm{HT}_{2}$ cascade; $n=$ $4 ; p=0.04)$.

Having established that RDE involves closure of strychninesensitive GlyRs and that the postsynaptic input conductance drops in response to RDE induction, we hypothesized that the crucial event underlying RDE is the closure of postsynaptic GlyRs that shunt EPSPs, with no change in presynaptic release of glutamate. In this case, one would expect RDE to be less pronounced under voltage clamp, which under the assumption of good space clamp should eliminate shunting effects. In concordance with this hypothesis, we found that RDE does not occur when the cell is voltage clamped (paired $t$ test, $p=0.97 ; n=6$; AP-EPSC pairs at $10 \mathrm{~Hz}$ vs EPSC alone at $10 \mathrm{~Hz}$ ). Additionally, we performed a $\mathrm{CV}$ analysis. This test measures changes in the presynaptic release probability, and therefore a change in the CV measure would implicate presynaptic receptors. We performed a CV analysis for EPSPs alone in control solutions, EPSPs alone in strychnine, and $10 \mathrm{~Hz}$ AP-EPSP pairs in control solutions and found no significant change between these groups (ANOVA: $p=0.21 ; F=1.62$; $\mathrm{df}=2$ ). These data imply that neither strychnine nor induction of RDE alters release probability.

We also examined the effects of strychnine on three additional measures of presynaptic release or postsynaptic input resistance: paired-pulse facilitation (a common test for presynaptic effects), excitability (a measure of the strength of the postsynaptic response to a depolarizing input, which is an indicator of postsynaptic changes), and AP threshold (a test for postsynaptic effects). We found that bath-applied strychnine has no effect on pairedpulse facilitation, as shown for a sample trace in Figure $4 a$ and the averages for a single cell in Figure $4 b$. Across a population of seven cells, we found that strychnine has no effect on paired-pulse facilitation (Fig. 4e). Bath-applied strychnine did, however, increase postsynaptic excitability and decrease the AP threshold, as is shown in a sample trace (Fig. $4 c$ ) and with averages for a single cell (Fig. $4 d$ ). Across a population of seven cells, we found that the mean excitability significantly increases (Fig. 4e) and that the mean AP threshold significantly decreases (Fig. 4e) with the application of strychnine. Together, these findings suggest the involvement of postsynaptic GlyRs in RDE.

\section{Pharmacological manipulation of RDE}

Because the application of extracellular glycine blocks RDE, effectively rendering postsynaptic GlyRs insensitive to AP-EPSP pairs, we reasoned that we might be able to manipulate baseline peak EPSP magnitude and/or RDE by inhibiting glycinergic transport. Extracellular glycine is transported by two classes of molecule: GlyT1, expressed mainly by glial cells (Zafra et al., 1995); and GlyT2, expressed mainly in glycinergic presynaptic terminals (Zafra et al., 1995; Danglot et al., 2004). In the presence of the GlyT1 antagonist ALX-5407 (500 nM), RDE persisted (paired $t$ test, $p=0.02 ; n=8$; AP-EPSP pairs at $10 \mathrm{~Hz}$ vs EPSP alone at $10 \mathrm{~Hz}$ ), and the EPSP alone did not change significantly from control conditions (paired $t$ test, $p=0.26 ; n=8$; EPSP alone in control solution vs EPSP alone in ALX-5407). In contrast, the application of the GlyT2 inhibitor amoxapine (100 $\mu \mathrm{M})$ led to effects that were similar to those induced by bath-applied glycine. In amoxapine, the magnitudes of EPSPs alone decreased, but this trend was nonsignificant (normalized value, $0.95 \pm 0.07$; $p=0.5$ ). Peak EPSP magnitudes measured during $10 \mathrm{~Hz} \mathrm{AP}-$
EPSP pairs decreased to the same magnitude as AP-EPSP pairs at $0.5 \mathrm{~Hz}$, as shown for an example cell in Figure $5 a$. Across a population of 12 cells, the mean peak EPSP magnitude measured for $10 \mathrm{~Hz}$ AP-EPSP pairs was reduced to that of the EPSP alone at 10 $\mathrm{Hz}$ (Fig. 5b). Amoxapine had no effect on synaptic facilitation (Fig. 5b, filled bars at low and high rates). As we observed for strychnine alone (Fig. 3c), coapplication of strychnine and amoxapine occludes RDE, such that the mean peak EPSP magnitude arising from EPSPs alone at $10 \mathrm{~Hz}$ is increased to the same magnitude measured for AP-EPSP pairs at $10 \mathrm{~Hz}(p>0.7)$ (Fig. $5 b)$.

We also examined effects of pharmacological manipulations and RDE induction protocols on input conductance. Bathapplied amoxapine did not change the postsynaptic membrane conductance (Fig. 5c). Interestingly, the coapplication of amoxapine (GlyT2 blocker) with ALX-5407 (GlyT1 blocker) does lead to an increase in the input conductance (ACSF with $1 \mathrm{~mm}$ glycine in the bath: normalized conductance control was $1.1 \pm 0.08$; normalized conductance with amoxapine, ALX-5407, and $1 \mathrm{~mm}$ glycine was $1.36 \pm 0.1 ; p=0.05 ; n=4)$. The decrease in conductance observed immediately after $10 \mathrm{~Hz}$ AP-EPSP pairs in control solution (Fig. $3 d$ ) is absent in the presence of amoxapine (Fig. $5 c$ ). As shown in Figure $5 c$, strychnine blocks open GlyRs and lowers input conductance by the same amount, independent of the presence or absence of amoxapine (Fig. $5 c$ ) ( $t$ test, $p>0.7$; input conductance change in strychnine with vs without amoxapine). Together with previous data, these results suggest that a subset of the postsynaptic GlyRs are open at rest but close in response to RDE induction. Inhibition of GlyT2-mediated reuptake (Fig. 5) or application of exogenous glycine (Fig. 3) blocks RDE, presumably by increasing external concentrations of glycine enough to overwhelm the RDE mechanism.

Having established that RDE is dependent on a reduction in the glycinergic conductance, we next investigated the mechanisms underlying RDE. This conductance could be reduced in two ways: (1) through a reduction in the extracellular glycine concentration (either through increased glycine transport out of the extracellular space or through a reduction in presynaptic glycine release) or (2) through a decrease in the GlyR response to glycine (either through decreased receptor sensitivity or through trafficking of the receptors out of the membrane). Seeking to distinguish between these two possibilities, we used a picospritzer to apply glycine ( $300 \mu \mathrm{M} ; 3 \mathrm{psi} ; 50-300 \mathrm{~ms}$, adjusted for current size in each cell) directly to the proximal apical dendrite of the whole-cell patch-clamped cell. As described previously (Chattipakorn and McMahon, 2002), locally applied glycine consistently led to a glycine-evoked current (Fig. $5 d$ ), which we measured in three conditions: (1) in the resting cell (control); (2) 50 ms after $10 \mathrm{~Hz}$ AP-EPSP pairs (i.e., during RDE); and (3) $50 \mathrm{~ms}$ after $1 \mathrm{~Hz}$ AP-EPSP pairs. If RDE were attributable to a decrease in the extracellular glycine concentration, we would expect an increase in the response to the glycine application after RDE induction compared with control because the lower glycine concentration would mean fewer GlyRs were open at that time (vs control), and therefore more GlyRs would open in response to the glycine application. If the GlyR response to glycine were decreased (through changes to the receptors), we would expect to measure a reduction in the response to applied glycine after RDE induction.

We measured a significant decrease in the magnitude of the response to the applied glycine after $10 \mathrm{~Hz}$ AP-EPSP pairs, compared with control, as shown in an example trace in Figure $5 d$. In all nine cells from which we recorded, the response to locally applied glycine significantly decreased after $10 \mathrm{~Hz}$ AP-EPSP 
a in control

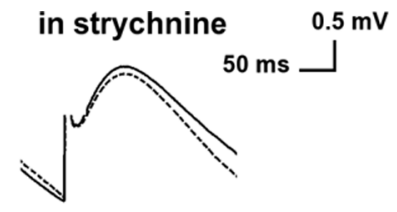

b

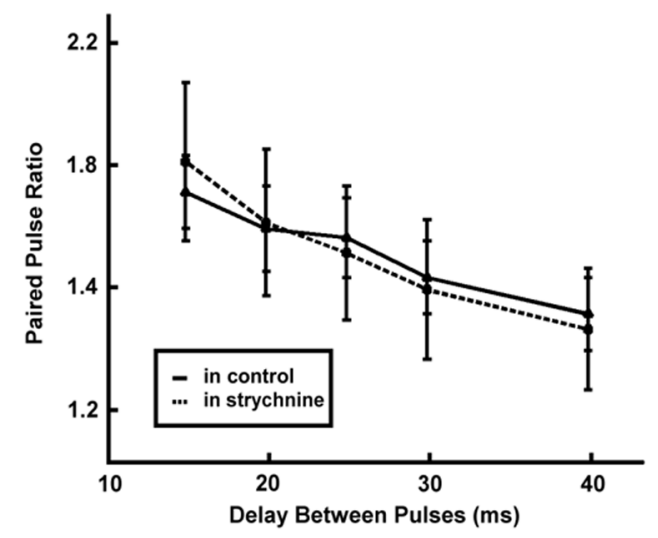

C in control

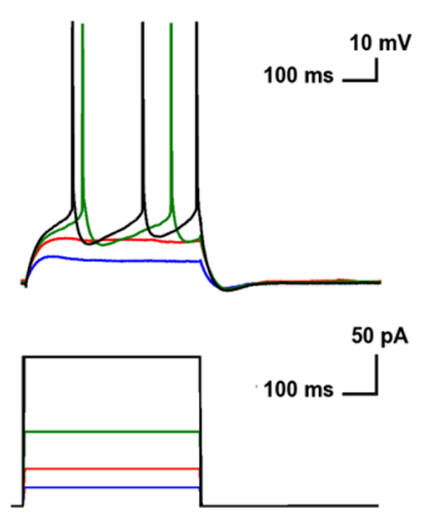

in strychnine

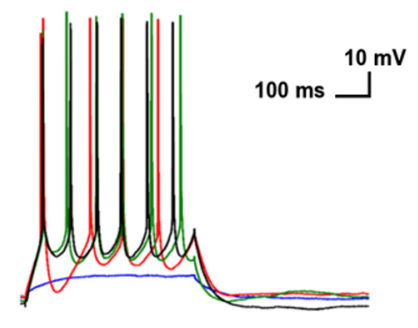

$100 \mathrm{~ms}$ d

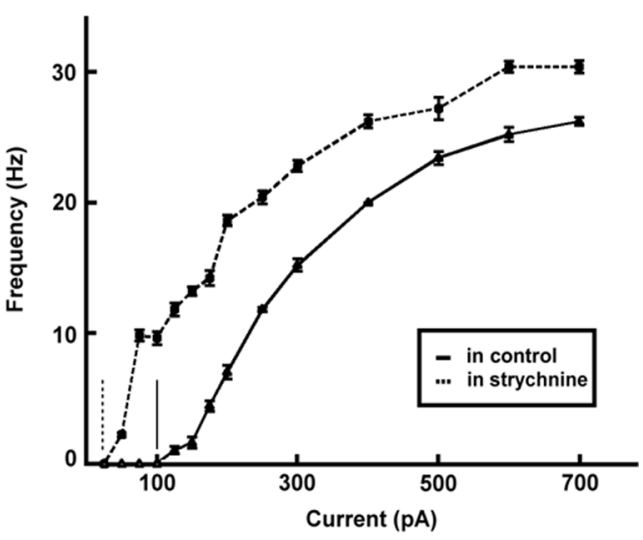

e

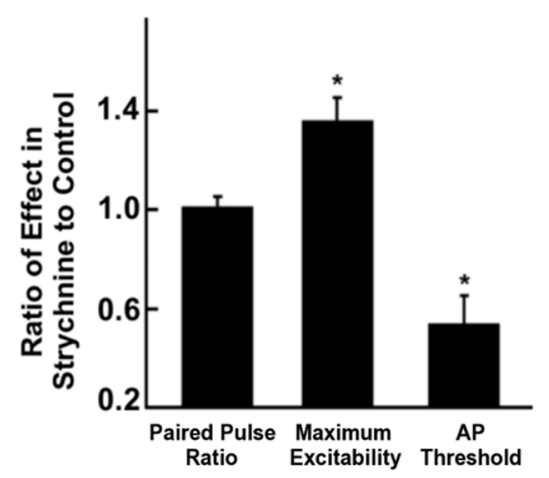

Figure 4. GlyRs involved in RDE are located postsynaptically. $\boldsymbol{a}$, Sample trace for paired-pulse facilitation in control (left) and strychnine (right). The second EPSP (solid line) is superimposed on the first EPSP (dashed line) to demonstrate the facilitation. $\boldsymbol{b}$, Single-cell measurements of paired-pulse ratio (PPR). The PPR (ratio of the magnitude of the second EPSP to the first EPSP) is plotted as a function of the delay between the two pulses. The PPRs in control solution (solid line) are not significantly different from the PPRs in strychnine (dashed line) (paired $t$ test at each delay, $p>$ 0.05). In this example, we observed facilitation in both ACSF and strychnine (1 $\mu \mathrm{M})$.c, Sample traces of excitability measurement in control (left) and strychnine (right; $1 \mu \mathrm{m} \mathrm{strychnine).} \mathrm{A} \mathrm{subset}$ of the voltage responses (top) to current injections (bottom) is shown. $\boldsymbol{d}$, Single-cell measurement of excitability. The firing rate is measured as a result of the current input. All frequencies recorded in strychnine (dashed line) are significantly different from the frequencies recorded for the same current input in control solution (solid line) (paired $t$ test at each input current, $p<0.05$ ), except where the current is $25 \mathrm{pA}$, which is below threshold in both solutions (paired $t$ test, $p>0.05$ ). AP threshold, marked by vertical lines on the $x$-axis, also decreases in strychnine (dashed line) compared with control (solid line). $e$, Averages of PPR, excitability, and AP threshold. The bars show the value measured in strychnine (1 $\mu \mathrm{M})$ normalized by the value measured in control ACSF. For excitability and AP threshold, but not for the PPR, the measurement made in control solution is significantly different from the measurement made in strychnine. For the PPR, we compared results at a delay of $15 \mathrm{~ms}$. For excitability, we compared the maximum frequency elicited for each cell. Paired $t$ test: PPR, $p=0.89, n=7$; excitability: $p=0.011, n=7 ; \mathrm{AP}$ threshold: $p=0.02, n=$ 4 (all comparisons were made between the same measurement in control solutions vs strychnine). Data are mean \pm SEM. ${ }^{*} p<0.05$.

pairs, compared with control (Fig. $5 e$, filled circles), with a decrease in the mean glycine-evoked response (normalized value, $0.85 \pm 0.04 ; p=0.00001 ; n=9)$. In contrast, responses to glycine application after $1 \mathrm{~Hz}$ AP-EPSP pairs were not significantly different from control (and were significantly larger than responses after $10 \mathrm{~Hz}$ AP-EPSP pairs) (Fig. $5 e$, open circles), with no change in the mean glycine-evoked response (normalized value, $0.97 \pm$ $0.03 ; p>0.8 ; n=9)$. These data suggest that during RDE, GlyR responsiveness is decreased, indicating that $\mathrm{RDE}$ is mediated, at least partially, through changes in the sensitivity or number of available GlyRs.

Next, we sought to determine whether postsynaptic $\mathrm{Ca}^{2+}$ plays a role in RDE. High (20 mM) intracellular concentrations of the calcium buffer BAPTA eliminated RDE, such that the mean magnitude of the EPSP alone at $10 \mathrm{~Hz}$ is not significantly different from that of AP-EPSP pairs at $10 \mathrm{~Hz}$ (Fig. 5b). These results suggest that the second-messenger cascade triggering RDE may be calcium mediated or could be calcium alone. With intracellular fluid that included $20 \mathrm{~mm}$ BAPTA, we found that the change in conductance induced by strychnine is not different from the conductance change induced by strychnine with control intracellular fluid ( $t$ test, $p=0.64 ; n=5$; data not shown), suggesting that the BAPTA alone does not change the glycinergic conductance. Together, our data suggest that the effects of BAPTA are like those of 
a

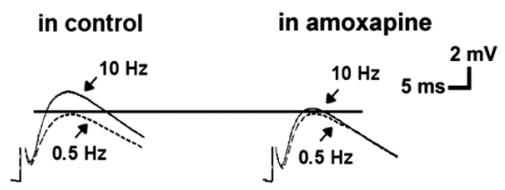

b

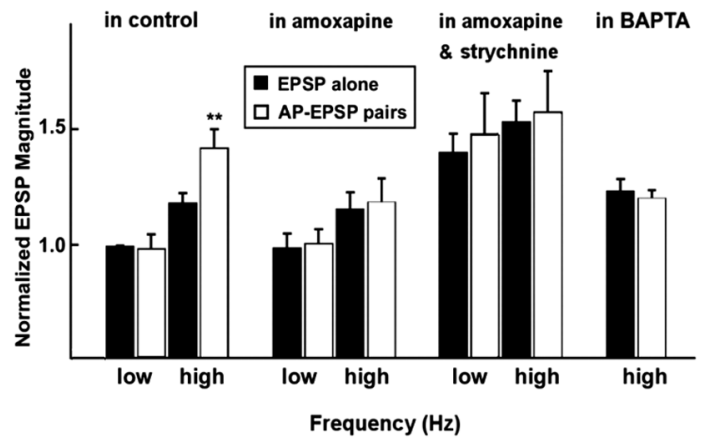

C

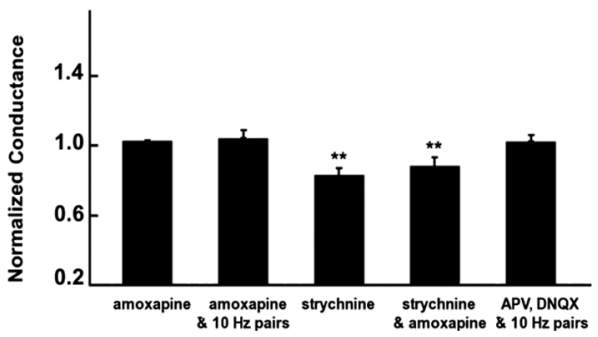

d

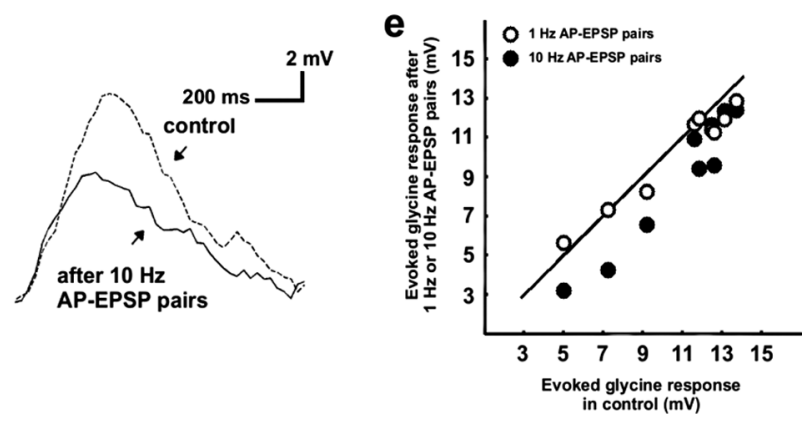

Figure 5. The GlyT2 inhibitor amoxapine blocks RDE, and GlyRs are less responsive during RDE. $\boldsymbol{a}$, Amoxapine prevents RDE. EPSPs are shown in control solution (left) and when the GlyT2 inhibitor (amoxapine, $100 \mu \mathrm{M}$ ) is bath applied (right). In amoxapine, the EPSP at high frequency (solid line) is the same size as the EPSP at low frequency (dashed line; all traces are from AP-EPSP pairs; APs not shown). $\boldsymbol{b}$, Normalized peak EPSP magnitudes for AP-EPSP pairs (open bars) and EPSPs alone (filled bars) as a function of both the bath solutions and the firing rate. AP-EPSP pairs at high frequency are significantly different from EPSPs alone at high frequency in control solution (Bonferroni's multiple comparison test, $p=3 \times 10^{-6} ; n=24$ ), but not in amoxapine (100 $\mu \mathrm{m}$; Bonferroni's multiple comparison test, $p=0.70 ; n=12$ ), amoxapine (100 $\mu \mathrm{M}$ ) plus strychnine ( $1 \mu \mathrm{m}$; Bonferroni's multiple comparison test, $p=0.72 ; n=4)$, or BAPTA (20 mm; paired $t$ test, $p=0.67 ; n=10$ ). AP-EPSP pairs at low frequency are not significantly different from EPSPs alone at low frequency in any solutions (Bonferroni's multiple comparison test: control solution, $p=0.64, n=24$; amoxapine, $p=0.22, n=12$; amoxapine plus strychnine, $p=0.20, n=4$ ). c, Conductance measured in the labeled solutions, normalized by the conductance measured in control solution. The glycinergic conductances in amoxapine are not significantly different from conductance measured in control solutions (paired $t$ test, $p>0.5 ; n=6$ ). Immediately after $10 \mathrm{~Hz}$ AP-EPSP pairs in the presence of amoxapine, conductance remains at control levels (conductance during pairing with bath-applied amoxapine vs control conductance; paired $t$ test, $p>0.05 ; n=12$ ). However, the addition of both strychnine and amoxapine decreases the glycinergic conductance compared with control solutions (paired $t$ test, $p<0.01 ; n=4$ ) in a manner that is not different from the affects of strychnine alone ( $p>0.7 ; n=12$ ). Glutamate blockers (50 $\mu \mathrm{m} \mathrm{APV} \mathrm{and} 10 \mu \mathrm{m}$ DNQX) prevent the glycinergic conductance change associated with $10 \mathrm{~Hz}$ AP-EPSP pairs ( $t$ test, $p=4.85 \times$ $10^{-7} ; 10 \mathrm{~Hz}$ AP-EPSP conductance change in control vs in APV and DNQX). Data in $\boldsymbol{B}$ and $\boldsymbol{C}$ are bath-applied amoxapine or exogenous glycine: a proportion of GlyRs are open and no longer close in response to high-frequency trains of AP-EPSP pairs. See the discussion for caveats associated with this interpretation.

Although we have demonstrated that RDE persists when either NMDA or AMPA receptors are blocked, we also blocked both NMDA and AMPA receptors using APV $(20 \mu \mathrm{M})$ and DNQX $(10 \mu \mathrm{M})$. We found that when pairing postsynaptic and presynaptic activity and measuring cellular input conductance as a surrogate for RDE (because there are no measurable EPSPs), we saw no change in input conductance (Fig. 5c), suggesting that RDE does not occur when both NMDA and AMPA receptors are blocked. This result suggests that the ionotropic effects of glutamate on either PCs or interneurons are important for RDE.

\section{RDE and LTD}

Our RDE induction protocol (repeated presentations of paired activity, with postsynaptic spikes leading presynaptic spikes by $10-60 \mathrm{~ms}$ ) is reminiscent of protocols that induce spike-timingdependent LTD in neocortex and entorhinal cortex (Sjostrom et al., 2001; Zhou et al., 2005) and timing-independent LTD at the CA3-CA1 synapse (Wittenberg and Wang, 2006). Although our internal controls demonstrated that RDE induction does not lead to LTD, we wanted to ask whether more repetitions of paired activity might give rise to LTD and whether induced LTD might depend on glycinergic metabolism. We focused on a single timing difference that induces LTD at this synapse (Wittenberg and Wang, 2006), in which the postsynaptic AP leads the presynaptic stimulation by $20 \mathrm{~ms}$. We first measured the frequency dependence of a spike-time-dependent plasticity-LTD protocol. We found strong effects of pairing frequency on LTD: when CPs were repeated at $10 \mathrm{~Hz}$ (Fig. 6b,c, blue) (average depression, $0.56 \pm$ 0.006 ), as shown in a sample trace in Figure $6 a$, we observed significant depression. When $\mathrm{CPs}$ were repeated at $1 \mathrm{~Hz}$, however, no significant depression was observed (Fig. 6b,c, black) (average depression, $1.02 \pm 0.007 ; t$ test, $p=0.0004 ; n=5$; depression at $1 \mathrm{~Hz}$ vs at $10 \mathrm{~Hz}$ ). To determine whether these effects are related to RDE, we measured LTD in the presence of strychnine, amoxapine, and high levels of glycine. In strychnine, even low-frequency $(1 \mathrm{~Hz}) \mathrm{CPs}$ led to LTD (Fig. 6b,c, green) (average depression, $0.62 \pm 0.006)$. In contrast, even in response to high-frequency $(10 \mathrm{~Hz}) \mathrm{CPs}$, both amoxapine (Fig. $6 b, c$, pink) (average depression, $0.99 \pm 0.006$ ) and high levels of glycine (Fig. $6 c$, white bars) (average depression, $1.1 \pm 0.01$ ) prevented LTD. This LTD is dependent on NMDA channels, because it is blocked by the bath application of APV (Fig. $6 c$, white bars) (average depression, $1.02 \pm 0.009)$ and the coapplication of APV and strychnine (Fig. $6 c$, white bars) (average depression, $1.01 \pm$ 0.006). Baseline peak EPSP magnitude before the conditioning stimulus and the subsequent amount of depression were uncorrelated in control solution (correlation: $r=-0.04$; $\mathrm{df}=8 ; p=$ 0.91). In contrast, the ratio of peak EPSP magnitude during CPs

$\leftarrow$

mean \pm SEM. ${ }^{* *} p<0.01$.d, Sample trace of response to locally applied glycine ( $300 \mu \mathrm{M}, 3 \mathrm{psi}$, $300 \mathrm{~ms}$; using a picospritzer). Response after $10 \mathrm{~Hz}$ AP-EPSP pairs (solid line) is reduced compared with the response in the resting cell (dashed line; control). $\boldsymbol{e}$, Peak amplitude in response to locally applied glycine (300 $\mu \mathrm{m}, 3 \mathrm{psi}, 50-300 \mathrm{~ms}$; using a picospritzer) in a resting cell (control) versus peak response amplitude $50 \mathrm{~ms}$ after $10 \mathrm{~Hz}$ AP-EPSP pairs (filled circles) or $1 \mathrm{~Hz}$ AP-EPSP pairs (open circles). There is a significant decrease in the glycine-evoked response after $10 \mathrm{~Hz}$ AP-EPSP pairs (Bonferroni's multiple comparison test; control vs $10 \mathrm{~Hz}$ AP-EPSP pairs, $p=0.0001$; control vs $1 \mathrm{~Hz}$ AP-EPSP pairs, $p>0.8 ; 10 \mathrm{~Hz}$ vs $1 \mathrm{~Hz}$ AP-EPSP pairs, $p=$ $0.001 ; n=9$ for all conditions). 

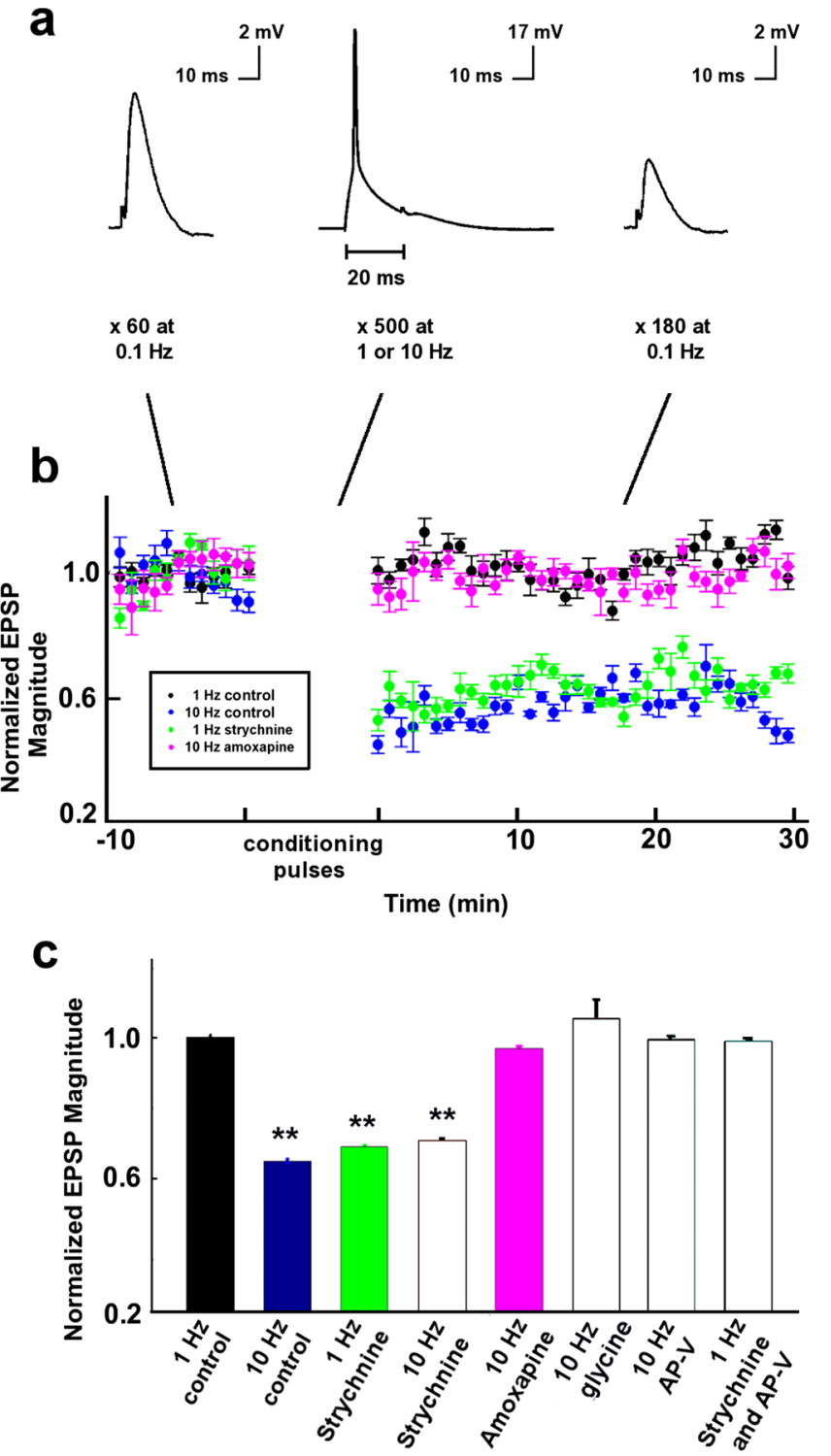

Figure 6. Long-term depression increases with the reduction of glycine suppression. $\boldsymbol{a}$ Sample traces of EPSPs 5 min before (left) and 10 min after (right) $10 \mathrm{~Hz}$ CPs (middle) in control solutions. $\boldsymbol{b}$, Peak EPSP magnitudes for before ( $-10 \mathrm{~min}$ to $\mathrm{CPs}$ ) and after (CPs to $30 \mathrm{~min})$ CPs. CPs were repeated at $1 \mathrm{~Hz}$ (black), $10 \mathrm{~Hz}$ (blue), $1 \mathrm{~Hz}$ in strychnine (10 $\mu \mathrm{m}$; green), and $10 \mathrm{~Hz}$ in amoxapine (100 $\mu \mathrm{m}$; pink) ( $n=5$ cells for each condition; average at each time point is shown). All experiments were recorded with bath-applied bicuculline. c, Average amount of depression from baseline (baseline mean of 1 ) for each of the aforementioned cases ( $n=5$ cells for each condition; 180 measurements per cell), and $10 \mathrm{~Hz}$ in strychnine (white bar), $10 \mathrm{~Hz}$ in glycine (1 $\mathrm{mm}$; white bar), $10 \mathrm{~Hz}$ in APV (50 $\mu \mathrm{m}$; white bar), and $1 \mathrm{~Hz}$ in strychnine (10 $\mu \mathrm{M}$ ) and APV (50 $\mu \mathrm{m}$; white bar). We find that the data fall into two groups: one that experienced significant depression ${ }^{* *} p<0.01$; $t$ test for post-CP peak EPSP magnitude vs baseline peak EPSP magnitude; $10 \mathrm{~Hz}$ control solution, $p=0.0008 ; 10 \mathrm{~Hz}$ strychnine, $p=0.01 ; 1 \mathrm{~Hz}$ strychnine, $p=$ 0.0007 ) and one that experienced little depression ( $t$ test for post-CP peak EPSP magnitude vs baseline peak EPSP magnitude; $1 \mathrm{~Hz}$ control solution, $p=0.48 ; 10 \mathrm{~Hz}$ amoxapine, $p=0.65 ; 10$ Hz glycine, $p=0.5 ; 10 \mathrm{~Hz}$ APV, $p=0.85 ; 1 \mathrm{~Hz}$ APV and strychnine, $p=0.70$ ). Data within a single group are statistically different (Bonferroni's multiple comparison test, $p<0.01$ ) from all comparisons made with the other group but are not significantly different (Bonferroni's multiple comparison test, $p>0.05$ ) within the same group.

relative to baseline magnitude was strongly correlated with the amount of observed LTD (correlation: $r=-0.7$; $\mathrm{df}=8 ; p=0.008$; the negative value of $r$ indicates that larger normalized EPSPs lead to more LTD). These results imply that RDE can affect LTD induced by repeated pairing of presynaptic and postsynaptic activity.

\section{Discussion}

Our results demonstrate a previously undetected form of modulation of glycinergic transmission in the hippocampus. As reported in previous work (Song et al., 2006), we found that a subset of GlyRs expressed by CA1 PCs are constitutively open in the hippocampal brain slice, creating a state of tonic inhibition. This inhibition is modulated by the rate of paired postsynaptic and presynaptic activity at the CA3-CA1 synapse. When the rate of paired APs and EPSPs increases to $5-10 \mathrm{~Hz}$, the glycinergic inhibition is reduced, giving rise to an increase in the peak EPSP magnitude in a phenomenon we refer to as RDE. RDE stands in stark contrast to traditional short-term synaptic facilitation, for two reasons. First, the locus of RDE is postsynaptic, whereas the locus of synaptic facilitation is typically presynaptic. Second, RDE requires correlated presynaptic and postsynaptic activity, whereas synaptic facilitation depends only on presynaptic activity.

RDE is blocked by exogenous glycine and is occluded by the GlyR antagonist strychnine. Additionally, RDE decreases the responsiveness of the GlyRs to local pressure-applied glycine. In response to longer trains of paired stimuli, we obtained robust LTD at stimulation frequencies that induce RDE, but not at lower rates that do not induce RDE. Pharmacological manipulations that block or occlude RDE lead to predictable changes in LTD, suggesting that RDE contributes to LTD induced by AP-EPSP pairs at rates of $\sim 10 \mathrm{~Hz}$.

\section{The mechanistic basis of RDE}

Several lines of evidence suggest that RDE is caused by the closure of postsynaptic GlyRs, a percentage of which are open in the quiescent slice. In the resting slice, $1 \mu \mathrm{M}$ strychnine decreases input conductance by $>15 \%$, and $10 \mathrm{~mm}$ exogenous glycine increases the input conductance by $>15 \%$. RDE is accompanied by a decrease in input conductance (or equivalently, an increase in input resistance) that is blocked by exogenous glycine and occluded by strychnine. Indicators of presynaptic release probability, including CV and paired-pulse facilitation, are unaltered by strychnine application, again suggesting that the relevant GlyRs are purely postsynaptic. High postsynaptic concentrations of BAPTA block RDE. Because changes in peak EPSP magnitude and membrane conductance after RDE quantitatively match those associated with strychnine, and because other obvious $\mathrm{Ca}^{2+}$-dependent mechanisms (e.g., $\mathrm{Ca}^{2+}$-activated $\mathrm{K}^{+}$channels) would lead to an increase (not a decrease/lack of change, as we observe) in both input conductance and peak EPSP magnitude because blocking calcium would close hyperpolarizing $\mathrm{K}^{+}$ channels, we believe that the closure of GlyRs is likely the dominant factor in RDE.

We hypothesize that RDE occurs because the closure of GlyR receptors during high-frequency AP-EPSP pairs reduces the dendritic shunt caused by the open receptors at rest. This hypothesis is critically dependent on the location of the GlyRs. The receptors are abundant in stratum pyramidale and proximal stratum radiatum (Danglot et al., 2004), a location through which excitatory signals would pass, and therefore be affected by the shunt from these receptors. The chloride Nernst potential measured in CA1 PCs at this age is approximately $-60 \mathrm{mV}$ (Xu and Sastry, 2007), consistent with a shunting role.

What causes the postsynaptic GlyRs to close? We suggest four possibilities: (1) a reduction in sensitivity of GlyRs, such that the GlyRs have a lower affinity for free glycine and are less likely to bind and open for a given local concentration of glycine; (2) trafficking of GlyRs out of the membrane during RDE, thereby 
reducing the number of available GlyRs; (3) an increase in the rate of glycine transport from the cleft into presynaptic terminals, which closes GlyRs by reducing the extracellular concentration of glycine at the synaptic cleft; or (4) a decrease in glycine release from presynaptic interneurons, also leading to a decrease in extracellular glycine concentrations near GlyRs.

We have shown that pressure-applied glycine evokes a smaller current during RDE. Assuming that local pressure application overwhelms any changes in transport, this result suggests that either model 1 (reduced GlyR sensitivity) or model 2 (trafficking GlyRs out of the membrane) must be a mechanism of RDE. Given that $\mathrm{RDE}$ has a rapid offset $(<100 \mathrm{~ms})$ and that bath-applied glycine prevents RDE, reduced GlyR sensitivity seems a more likely explanation than receptor trafficking. This result also suggests that model 3 and model 4 are likely not mechanisms of RDE, because we would expect to measure an increase in the glycineevoked response if the levels of extracellular glycine were reduced; however, we cannot rule out that RDE is caused by a combination of reduced GlyR responsiveness and a reduction in the extracellular levels of glycine.

We also found that inhibiting GlyT2 reuptake with amoxapine prevents RDE from occurring. This result suggests that upregulation of transport (model 3 ) at presynaptic glycinergic terminals may also contribute to RDE. Another possibility is that amoxapine exerts its effects simply by increasing the concentration of glycine near synaptic GlyRs, driving synaptic GlyRs closer to saturation and thus making RDE-induced changes in receptor sensitivity harder to detect. The fact that amoxapine prevents RDE without changing input conductance suggests the possibility that synaptic GlyRs are preferentially responsible for RDE, whereas extrasynaptic GlyRs (Danglot et al., 2004) may contribute more to changes in input conductance as measured from the soma. In any event, we find the results supporting decreased GlyR sensitivity or number more direct and thus more compelling.

\section{Convergence of presynaptic and postsynaptic signals}

As we have noted, a hallmark feature of RDE is that signals resulting from presynaptic and postsynaptic activity must converge to induce it. We do not yet know the site of convergence. Models of convergence involving reduction in glycinergic release would seem to be ruled out by the fact that RDE suppresses responses to pressure-ejected glycine. Viable models include convergence within the postsynaptic CA1 PC, leading to either a reduction in GlyR sensitivity or increased GlyR trafficking away from the postsynaptic site. The fact that $20 \mathrm{~mm}$ BAPTA blocks RDE suggests that postsynaptic $\mathrm{Ca}^{2+}$ may be involved; however, BAPTA would also block release from the postsynaptic cell, leaving open the possibility that effects of feedback from the PC is involved. If $\mathrm{Ca}^{2+}$ is an important second messenger, we can draw some conclusions. First, because RDE persists in the presence of APV, postsynaptic NMDA receptors cannot be the point of convergence. Second, because buffering calcium does not alter the glycinergic conductance, any important postsynaptic calcium signal is likely to be upstream of the point of convergence. Sensitivity of RDE to coapplication of APV and DNQX suggests that feedforward or feedback ionotropic excitation may play a role.

\section{RDE and LTD}

Our results demonstrate that LTD with so-called "anticausal" pairing (postsynaptic AP $20 \mathrm{~ms}$ before the EPSP) depends strongly on the frequency of pairing. Specifically, we see little LTD in response to $1 \mathrm{~Hz}$ pairs but robust LTD with $10-\mathrm{Hz}$ pairing. Three pieces of evidence suggest that RDE may contribute to the frequency-dependent LTD we observed. First, these two induction frequencies, respectively, lie below and above the frequency threshold for RDE. Second, strychnine occludes RDE and leads to high-frequency outcomes during LTD induction even with low-frequency induction protocols. Third, amoxapine and exogenous glycine block both RDE and LTD, giving lowfrequency outcomes even at high frequencies. Although we find these correlations striking, we do not want to overinterpret this result: a large number of factors, no doubt, contribute to the dependence of LTD on frequency and other experimental parameters. Furthermore, glycinergic metabolism is likely to also participate in NMDA-dependent LTD and LTP via its role as a coagonist for the NMDA receptor (Bergeron et al., 1998; Tsai et al., 2004; Martina et al., 2005).

Our finding of minimal LTD at low induction rates contrasts somewhat with previous findings, in which mild (Wittenberg and Wang, 2006) or robust (Normann et al., 2000) LTD is induced by similar low-frequency protocols at this synapse. The contrast among these results may relate to differences in pharmacological conditions. Wittenberg and Wang (2006) did the anticausal recordings without blocking inhibition (whereas we blocked GABAergic inhibition using bicuculline). Normann et al. (2000) blocked inhibition, but used picrotoxin. Given our body of results, we speculate that they may have seen robust LTD at low pairing rates because they inadvertently blocked GlyRs.

\section{Future studies}

Our results provide a foundation for understanding how correlated presynaptic and postsynaptic activity leads to closure of postsynaptic GlyRs and consequent enhancement of peak EPSP magnitude. Further study is necessary to determine the roles of postsynaptic $\mathrm{Ca}^{2+}$ and other potential second messengers in RDE. We showed that RDE is unaffected by antagonists of CB1, nitric oxide, and the $5-\mathrm{HT}_{2}$ cascade. Other possible candidates include endogenous cannabinoids anandamide (AEA) and 2-AG, which are known to block GlyRs (Lozovaya et al., 2005), and AEA, which also directly upregulates glycine transport (Pearlman et al., 2003). It is important to note that our study was performed in animals aged P14-P21. At this age, the mature complement of chloride transporters that establish $E_{\mathrm{Cl}}$ is present (Rivera et al., 1999), but the animals are juvenile nonetheless. Although GlyRs persist in hippocampus through adulthood (Danglot et al., 2004), whether or not RDE is a developmental phenomenon requires further study.

Finally, our results suggest that there is a link between RDE and LTD, either because common mechanisms drive both phenomena or because RDE induction contributes directly to LTD. We speculate that induction of RDE during an LTD-inducing stimulus enhances postsynaptic calcium transients in less leaky dendrites. (We know of no evidence suggesting that GlyRs are expressed on the spines themselves.) We are unlikely to identify the mechanistic connection between RDE and LTD until we better understand the mechanisms of each.

\section{References}

Abbott LF, Regehr WG (2004) Synaptic computation. Nature 431:796-803. Bergeron R, Meyer TM, Coyle JT, Greene RW (1998) Modulation of N-methyl-D-aspartate receptor function by glycine transport. Proc Natl Acad Sci U S A 95:15730-15734.

Chattipakorn SC, McMahon LL (2002) Pharmacological characterization of glycine-gated chloride currents recorded in rat hippocampal slices. J Neurophysiol 87:1515-1525.

Danglot L, Rostaing P, Triller A, Bessis A (2004) Morphologically identified glycinergic synapses in the hippocampus. Mol Cell Neurosci 27:394-403. 
Hausser M, Major G, Stuart GJ (2001) Differential shunting of EPSPs by action potentials. Science 291:138-141.

Jursky F, Nelson N (1995) Localization of glycine neurotransmitter transporter (GLYT2) reveals correlation with the distribution of glycine receptor. J Neurochem 64:1026-1033.

Krause M, Jia Y (2005) Serotonergic modulation of carbachol-induced rhythmic activity in hippocampal slices. Neuropharmacology 48:381-390.

Li Y, Wu LJ, Legendre P, Xu TL (2003) Asymmetric cross-inhibition between GABAA and glycine receptors in rat spinal dorsal horn neurons. J Biol Chem 278:38637-38645.

Lozovaya N, Yatsenko N, Beketov A, Tsintsadze T, Burnashev N (2005) Glycine receptors in CNS neurons as a target for nonretrograde action of cannabinoids. J Neurosci 25:7499-7506.

Martina M, Gorfinkel Y, Halman S, Lowe JA, Periyalwar P, Schmidt CJ, Bergeron R (2004) Glycine transporter type 1 blockade changes NMDA receptor-mediated responses and LTP in hippocampal CA1 pyramidal cells by altering extracellular glycine levels. J Physiol 557:489-500.

Martina M, ME BT, Halman S, Tsai G, Tiberi M, Coyle JT, Bergeron R (2005) Reduced glycine transporter type 1 expression leads to major changes in glutamatergic neurotransmission of CA1 hippocampal neurones in mice. J Physiol 563:777-793.

Normann C, Peckys D, Schulze CH, Walden J, Jonas P, Bischofberger J (2000) Associative long-term depression in the hippocampus is dependent on postsynaptic N-type $\mathrm{Ca}^{2+}$ channels. J Neurosci 20:8290-8297.

Pearlman RJ, Aubrey KR, Vandenberg RJ (2003) Arachidonic acid and anandamide have opposite modulatory actions at the glycine transporter, GLYT1a. J Neurochem 84:592-601.

Pribilla I, Takagi T, Langosch D, Bormann J, Betz H (1992) The atypical M2 segment of the beta subunit confers picrotoxinin resistance to inhibitory glycine receptor channels. EMBO J 11:4305-4311.

Racca C, Gardiol A, Triller A (1998) Cell-specific dendritic localization of glycine receptor alpha subunit messenger RNAs. Neuroscience 84:997-1012.

Rivera C, Voipio J, Payne JA, Ruusuvuori E, Lahtinen H, Lamsa K, Pirvola U, Saarma M, Kaila K (1999) The K+/Cl- co-transporter KCC2 renders
GABA hyperpolarizing during neuronal maturation. Nature 397:251-255.

Roux MJ, Supplisson S (2000) Neuronal and glial glycine transporters have different stoichiometries. Neuron 25:373-383.

Sjostrom PJ, Turrigiano GG, Nelson SB (2001) Rate, timing, and cooperativity jointly determine cortical synaptic plasticity. Neuron 32:1149-1164.

Song W, Chattipakorn SC, McMahon LL (2006) Glycine-gated chloride channels depress synaptic transmission in rat hippocampus. J Neurophysiol 95:2366-2379.

Tsai G, Ralph-Williams RJ, Martina M, Bergeron R, Berger-Sweeney J, Dunham KS, Jiang Z, Caine SB, Coyle JT (2004) Gene knockout of glycine transporter 1: characterization of the behavioral phenotype. Proc Natl Acad Sci U S A 101:8485-8490.

Wittenberg GM, Wang SS (2006) Malleability of spike-timing-dependent plasticity at the CA3-CA1 synapse. J Neurosci 26:6610-6617.

Wojcik SM, Katsurabayashi S, Guillemin I, Friauf E, Rosenmund C, Brose N, Rhee JS (2006) A shared vesicular carrier allows synaptic corelease of GABA and glycine. Neuron 50:575-587.

Xu JY, Sastry BR (2007) Theta-bursts induce a shift in reversal potentials for GABA-A receptor-mediated postsynaptic currents in rat hippocampal CA1 neurons. Exp Neurol 204:836-839.

Zafra F, Aragon C, Olivares L, Danbolt NC, Gimenez C, Storm-Mathisen J (1995) Glycine transporters are differentially expressed among CNS cells. J Neurosci 15:3952-3969.

Zeilhofer HU, Studler B, Arabadzisz D, Schweizer C, Ahmadi S, Layh B, Bosl MR, Fritschy JM (2005) Glycinergic neurons expressing enhanced green fluorescent protein in bacterial artificial chromosome transgenic mice. J Comp Neurol 482:123-141.

Zhang LH, Xu L, Xu TL (2006) Glycine receptor activation regulates shortterm plasticity in CA1 area of hippocampal slices of rats. Biochem Biophys Res Commun 344:721-726.

Zhou YD, Acker CD, Netoff TI, Sen K, White JA (2005) Increasing Ca2+ transients by broadening postsynaptic action potentials enhances timingdependent synaptic depression. Proc Natl Acad Sci USA 102:19121-19125.

Zucker RS, Regehr WG (2002) Short-term synaptic plasticity. Annu Rev Physiol 64:355-405. 